\title{
Systematic Identification of the Cancer Pathways and Molecules Related With Breast Cancer Immunogenicity
}

\section{Xiaoli Wang}

Jiangsu Cancer Hospital; jiangsu institution of cancer research; the affiliated cancer hospital of Nanjing Medical University

Silu Xu

Jiangsu Cancer Hospital; Jiangsu institution of cancer research; the affiliated cancer hospital of Nanjing Medical University

\section{Lingli Huang}

Jiangsu Cancer Institute and Hospital: Jiangsu Cancer Hospital

\section{Lei Wang}

Jiangsu Cancer Hospital

Nan Wu ( $\nabla$ nan.wu@njmu.edu.cn )

Jiangsu Cancer Hospital;Jiangsu Institute of Cancer Research; the affiliated cancer hospital of Nanjing Medical University https://orcid.org/0000-0003-0942-5278

\section{Research Article}

Keywords: breast cancer, tumor immunity, immunotherapy, miRNA, PD-L1

Posted Date: February 17th, 2021

DOI: https://doi.org/10.21203/rs.3.rs-176051/v1

License: (c) (i) This work is licensed under a Creative Commons Attribution 4.0 International License. Read Full License 


\section{Abstract}

To identify molecular features related to immunogenic activity in breast cancer (BC) and provide new targets and directions for BC immunotherapy. We first used ESTIMATE to evaluate the degree of immune cell infiltration of the BC patients in TCGA and METABRIC, and explore the relationship between the degree of immune cell infiltration and prognosis of breast cancer patients. Then, we identified the cancer pathways, proteins, miRNAs related to BC immunogenicity, and predicted miRNAs target genes and identified the pathways related to target genes with KEGG pathway enrichment analysis. We also explored the correlation between PD-L1 expression level and cancer pathways and found that PD-L1 expression showed a positive association with cancer pathways. In this article we have successfully identified several cancer pathways, proteins, miRNAs and their target genes, which could be as promising new target for BC immunotherapy. And PD-L1 blockade therapy may be more effective in $\mathrm{BC}$ patients with the activation of some cancer pathways.

\section{Introduction}

For women, breast cancer (BC) is the most common cancer, as well as the second leading cause of cancer death in female population [1]. At present, drug treatment of BC is mainly restricted in chemotherapy $\triangle$ endocrine therapy and HER-2 targeted therapy. Immunotherapy has achieved notable progress in a variety of cancers including melanoma, non-small cell lung cancer, renal cell cancer, Hodgkin's lymphoma, bladder cancer, head and neck cancer [2]. However, immunotherapeutic strategies for breast cancer are still limited so far. Although BC is not highly responsive to immunotherapy when compared with other malignant disease like melanoma and non-small cell lung cancer, there is emerging evidence shows variable immunogenicity activity exists in certain BC subtypes [3]. Recently, for triple negative breast cancer (TNBC) which is highly invasive and lacking of treatment targeting hormone or HER2 receptor, Atezolizumab in combination with paclitaxel proteinbound has been accelerated approved by FDA for the treatment of adult patients with unresectable locally advanced or metastatic TNBC, continued approval might be contingent upon verification and description of clinical benefit in confirmatory trials [4]. This indicates a promising prospect for BC immunotherapy in future.

Concerning to immunotherapy, which has benefited only a subset of patients, with some patients failing to respond to treatment at all and others achieving a limited response followed by tumor progression [5], Identification of molecular biomarkers predicting tumor immunotherapy benefits would be crucial. There have been evidences indicating that PD-L1, dMMR, MSI-H, TMB and other biomarkers been related to tumor immunotherapy [6-9]. IHC positivity of PD-L1 in tumor cells or immune cells was predictive of better response of anti-PD-(L)1 therapy [7]. A phase 2 study conducted by Le et al [8] evaluated the clinical activity of pembrolizumab, the data from which indicated tumors with dMMR are more responsive to immunotherapy. Snyder et al [9] have found that high mutational load in melanoma correlated with sustained clinical benefit from ipilimumab or tremelimumab-based CTLA-4 inhibition. Therefore, it should be noted that the data supporting these biomarkers are mainly obtained from lung cancer, malignant melanoma and colorectal cancer, and data originating from BC cases are still quite limited. In addition, even as the most widely accepted biomarker, there are also up to $20 \%$ patients with PD-L1-negative tumors benefit from immune therapies [8,10-11]. This suggests that further screening of biomarkers for accurate prediction of breast cancer immunotherapy response is of extreme importance in the BC immunotherapy.

In this study, we successfully identified the cancer signaling pathways, proteins and miRNAs that are closely related to the immunogenicity of $\mathrm{BC}$, predicted the target genes regulated by these miRNAs, and explored the relationship between PD-L1 expression level and these pathways. Our findings provided new basis and direction for future immunotherapy of BC.

\section{Materials \& Methods}

\section{Data sources}

TCGA RNA-Seq gene expression profile (Level 3), protein expression profile (Level 3), miRNA expression data and clinical data of BC were acquired from Genomic Data Commons Data Portal (https://portal.gdc.cancer.gov/). METABRIC gene 
expression profile and clinical date were acquired from cBioPortal (http://www.cbioportal.org). Computing and statistical analysis were carried out by R (https://www.r-project.org/).

We analyzed the enrichment levels of 8 immune signatures based on gene expression profiles, as well as the enrichment levels of 23 cancer pathways screened using KEGG. The 8 immune signatures included CD8+ T cells, immune checkpoints, cytolytic activity, human leukocyte antigen (HLA), macrophages, natural killer (NK) cells, tumor infiltrating lymphocytes (TILs), and regulatory T cells (Treg). The 23 cancer pathways included NOD-like receptor, Toll-like receptor, JAK-STAT, MAPK, PI3K-AKT, apoptosis, Focal adhesion, Ca2+, Wnt, VEGF, TNF, HIF-1, Hedgehog, Notch, ECM-like receptor, NF-KB, TGF- $\beta$, glycolysis, ErbB, estrogen receptor, MMR, cell cycle and p53 signaling pathway.

\section{Activity-evaluation of immune Cell types and functions}

We used the sSGSEA score to quantify the activity or enrichment level of different immune cells, immune functions and cancer pathways in tumor samples. A higher sSGSEA score represents a higher activity. In addition, we used ESTIMATE algorithm to estimate the level of immune infiltration within tumor tissue [12]. Based on gene expression profile, ESTIMATE outputted an immune score for each sample to quantify the degree of immune cell infiltration in tumor tissue.

\section{Prediction of microRNA target gene}

Firstly, TargetScan 7.2 was used to obtain the target genes predicted by miRNA, then miRWalk 2.0, miRtarBase, miRmap and miRPathDB were used. The target genes simultaneously predicted by all the 5 databases were selected as the target genes regulated by miRNA. Finally, Cytoscape was used to construct the regulatory network between the target genes.

\section{Survival analysis}

Survival analysis of BC patients was performed based on immune score data. The Kaplan-Meier survival curve was used to represent the differences in OS and DFS between patients with high or low immune score. The median immune score was used to identify patients with high or low immune scores. The log-rank test was used to calculate the difference of survival time between the two groups of patients, and $P<0.05$ represented a significant difference.

\section{Statistics and analysis}

Pearson's correlation was used to calculate the correlation between immunity and the cancer pathway (ssGSEA score). Spearman's correlation was used to calculate the correlation between gene expression level and cancer pathway enrichment level, as well as the correlation between immune score and the level of cancer pathway enrichment, protein expression or miRNA expression. The error discovery rate (FDR) calculated using Benjamini and Hochberg (BH) methods was used to rectify the $P$ value. FDR $<0.05$ was considered statistically significant.

\section{Results}

\section{The degree of immune Cell infiltration is positively correlated with the survival prognosis of BC Patients}

In the TCGA and METABRIC data set, we compared the survival of BC patients with different level of immune cell infiltration degree (high or low immune score). Both of the outcomes of the two data sets showed that $\mathrm{BC}$ patients with high immune score had a significantly better overall survival (OS) (sequential test $P<0.05$, Fig. 1), which indicates that BC patient with high immunity infiltration has better clinical outcomes.

\section{The cancer-related pathway is positively correlated with the degree of immune Cell infiltration and immunogenicity}

Using KEGG pathway enrichment analysis, we identified 23 cancer pathways: NOD-like receptor, Toll-like receptor, JAK-STAT, MAPK, PI3K-AKT, apoptosis, Focal adhesion, Ca2+, Wnt, VEGF, HIF-1, TNF, Hedgehog, Notch, ECM-like receptor, NF-KB, TGF- $\beta$, 
glycolysis, ErbB, estrogen receptor, MMR, cell cycle, and p53 signaling pathways. We compared the relationship between cancer pathway activity and the infiltration degree of BC immune cells in TCGA and METABRIC data sets. The results showed that in the two data sets, almost all cancer pathways were positively correlated with the infiltration degree of immune cells, only Hedgehog and Notch were negatively correlated with the infiltration degree of immune cells (Spearman's correlation, FDR $<0.05$, Fig. 2a). Then we further compared the correlation between the activity of cancer pathway and the 8 immune characteristics. Being consistent with the degree of immune cell infiltration, almost all of the cancer pathways are positively correlated with the activity of the 8 immune characteristics, while Hedgehog and Notch were negatively associated with immune characteristics (Pearson's correlation, FDR $<0.05$, Fig. 2b).

\section{Identify key proteins associated with immune score and immunogenicity}

We compared the correlation between protein and immune cell infiltration at protein levels. Eight proteins were identified being positively correlated with the immunity score: LCK, cleaved_capase-7, Syk, Axl, PI3K, Annexin-1, PKC-a, ATM. Five proteins were negatively correlated with immune score: ER-a, E-Cadherin, GATA3, HER3, Claudin-7 (Spearman's correlation, |R| $>0.25$, FDR $<0.05$, Fig. 3a). In further analysis, the correlation between these proteins and the 8 immune characteristic activities were almost the same (Pearson's correlation, FDR $<0.05$, Fig. 3b). These results suggest that the expression of the up or down regulated key proteins we identified is associated with increase or decrease of immunogenicity in BC.

\section{The correlation between microRNA (miRNA) and immune score}

microRNA (miRNA) is a conserved noncoding RNA segment of 22-25 nucleotide sequences that regulates gene expression at the transcriptional level by targeting specific mRNAs and leading to their degradation or inhibition the translation. miRNAmediated gene regulation is critical to cell function, and more than one-third of mRNAs might be miRNA targets [13]. Recent studies have shown that miRNA also plays a key role in the regulation of immune function, including innate and adaptive immune responses, immune cell development and differentiation and autoimmunity prevention [14].

Spearman's correlation was used to compare the correlation between miRNA and the immune cell infiltration degree within TCGA. According to FDR < 0.05, 187 miRNAs positively correlated with immune score and 69 miRNAs negatively correlated with immune score were identified. By further setting $\mathrm{R}>0.5,4$ miRNAs positively correlated with immune score were identified: hsa-mir-155, hsa-mir-150, hsa-mir-146 and hsa-mir-223. By setting $|\mathrm{R}|>0.25,4$ miRNAs negatively correlated with immune score were identified: hsa-mir-96, hsa-mir-182, hsa-mir-125a, and hsa-mir-190b (Fig. 4).

We further used TargetScan 7.2 to predict target genes of the four miRNAs which had a correlation coefficient of immune score greater than 0.5 : hsa-mir-155, hsa-mir-150, hsa-mir-146a and hsa-mir-223. In order to reduce false positive rate and ensure the reliability, miRWalk 2.0, miRPathDB, miRTarBase and miRNAMap were simultaneously used for prediction. The intersection of above databases and software was taken and the results were shown in Table 1.

We further compared the correlation between these target genes and the infiltration degree of immune cells (Spearman's correlation, FDR < 0.05), and analyzed the regulation network of the selected target genes (Fig. 5a).

Finally, we performed KEGG pathway enrichment analysis on these genes. The results showed these genes were involved in multiple cancer-relating signaling pathways in BC, such as NOD-like receptor, Toll-like receptor, JAK-STAT, MAPK, apoptosis, Focal adhesion, Ca2+, Wnt, VEGF, Hedgehog, Notch, TGF- $\beta$, glycolytic, ErbB, cell cycle, and p53 signaling pathways. They also involved in several immune-relating pathways, such as B cell receptor signaling pathway, T cell receptor signaling pathway, chemokine signaling pathway, NK cell-mediated cytotoxicity signaling pathway, and cytokine-cytokine receptor interaction pathway (Fig. 5b). This indicates that miRNA plays an important regulatory role in BC as well as the immunotherapy of $\mathrm{BC}$, which may be achieved by regulating the target genes.

\section{Correlation between PD-L1 and cancer pathway activity}


The expression of PD-L1 on tumor cells plays a key role in tumor immune escape [15]. We analyzed the correlation between PD-L1 expression and cancer pathway activity. It was found that in the two data sets that the expression level of PD-L1 was significantly positively correlated with the activity of 11 cancer pathways (NOD-like receptor, Toll-like receptor, NF-KB, JAKSTAT, TNF, cell apoptosis, HIF-1, VEGF, ErbB, and p53 pathway), while negatively correlated with the activity of Notch pathway (Spearman's correlation, $P<0.05$ ) (Fig. 6). This reveals that PD-1 or PD-L1 blocking therapy may be more effective for breast cancer subtypes in which these cancer pathways were activated.

\section{Discussion}

In order to identify the molecular characteristics associated with immunogenicity of BC, we compared the survival of BC patients with different degree of immune cell infiltration. The results showed that BC patients with high immune invasion had better clinical prognosis.

Then we identified 23 common cancer pathways in BC from KEGG and analyzed the correlation between cancer pathway activity and the degree of immune cell infiltration. The correlation between cancer pathway activity and 8 immune characteristics were simultaneously analyzed. We found that almost all of these cancer pathways were positively correlated with the degree of immune cell infiltration, while Hedgehog signaling pathway and Notch signaling pathway were negatively correlated.

A research by Otsuka $A$ et al has shown that inhibition of the Hedgehog signaling pathway could change tumor microenvironment, transform the chemokine and cytokine network, increase the amount of CD8+ cytotoxic T cells invading tumor cells and promote adaptive immune response of basal cell carcinoma [16]. Notch signal negatively regulates the inflammatory response that activated by Toll-like receptor (TLR), an important molecule in nonspecific immunity, which plays a connecting role in nonspecific immunity and specific immunity [17]. Therefore, inhibition of Hedgehog or Notch pathway may enhance the efficacy of immunotherapy in BC. Thus the combination of Hedgehog or Notch pathway inhibitor and immunotherapy might be a potential direction for the treatment of breast cancer.

At the protein level, we identified 13 proteins that were most relevant to immune score and immune characteristics. Among them, 8 proteins were identified being positively correlated with the immunity score: LCK, cleaved_capase-7, Syk, Axl, PI3K, Annexin-1, PKC-a, ATM; while another 5 proteins were negatively correlated: ER-a, E-Cadherin, GATA3, HER3, Claudin-7. Among the 5 proteins, LCK is one of the molecules from protein tyrosine kinase (SRC) family that plays a key role in the $T$ cell receptor (TCR) signaling pathway. There had been study showing that the inhibitory effect of PD-1 on TCR signal molecule activation is significantly enhanced by PD-1 mediated LCK inhibition [18]. Capase-7 is a key protein in the cell apoptotic pathway, which regulates immune response by mediating inflammation [19]. As an adaptive immune receptor signal, the role of Syk is crucial in innate immune recognition [20]. Axl overexpresses in a variety of tumor tissues and cells. Wei et al [21] combined AxI with chimeric antigen receptor T cells, namely AXL-CAR-T, and found it exhibits obvious antitumor effect in TNBC xenotransplantation model, implying Axl could be a potential therapeutic target for TNBC [21]. Annexin-1, a significant anti-inflammatory protein controlling the interleukin-mediated immune response [22], has both innate and adaptive immune function and mainly participates in inflammatory cell regulation. As adherence junction protein, E-cadherin adjusts a number of intracellular signal transduction pathways. Recent studies have revealed that E-cadherin affects immunity via regulating mononuclear macrophages [23]. Down regulation of Claudin-7 induces metastasis and invasion of colorectal cancer by promoting epithelial-mesenchymal transition [24]. The zinc finger transcription factor GATA3, a major regulator of T helper 2 (Th2) cell differentiation, modulates the expression of IL-4, IL-5 and IL-13 [25]. GATA-3 facilitates type II immunity by adjusting the development and function of type 2 innate lymphocyte cells (ILC-2) [26]. HER3 is crucial heterodimer ligand of EGFR and HER2 and high expression of it accelerates the invasion of BC. Therefore, HER3 inhibition could be widely used in the treatment of EGFR- and HER2-driven tumors. Osada T et al [27] reported that HER3 targeted vaccine activates HER3 specific T cells and induces anti-HER3 specific antibodies, which changes both the T cell infiltration inside tumor and the response to immune checkpoint inhibition. Based on the expression of these up- or down- 
regulated protein molecules we identified is closely related to the increase or decrease of immunogenicity in $\mathrm{BC}$, we provided a direction for immunotherapy of $\mathrm{BC}$ at the protein level.

MicroRNA (miRNA) is a group of small ultra-conserved noncoding RNA that regulates gene expression at transcriptional level. They targets specific mRNA and consequently induce their degradation or inhibit their translation. By this way, miRNA plays a quite important role in the immune system, cell differentiation, tumorigenesis and cell death [28]. We identified miRNAs that are significantly associated with immunity through Spearman's analysis. The result indicated that the expression levels of these up- or down-regulated miRNAs are also related to the increase or decrease of immunogenicity in BC. It have been reported that the serum expression of miR-155 has increased more than 2 times in BC patients, which is positively correlated with the clinical stage and Ki-67 expression, while negatively correlated with p53 status [29]. It also regulates the production of mature B cells [13]. Another important miRNA, miR-150, targets at NOTCH3 and impacts both the physiology and development of T cell [30]. miR-146a inhibits the proliferation and metastasis of TNBC cells by regulating SOX5 [31], and also adjusts immune response in the microenvironment of melanoma [32]. miR-223 regulates immune response via adjusting granulocyte production and inhibiting of macrophage differentiation [33].

Subsequently, we predicted the target genes that regulated by the 4 miRNAs with a correlation coefficient greater than 0.5 through prediction software and database, and then analyzed the regulatory network and enrichment of KEGG pathway of these target genes. Regulatory network analysis showed these target genes had significant correlation with immunogenicity. KEGG pathway enrichment analysis showed that these genes participated in amount of BC relating signal pathways that identified by us, and also participated in several immune relating pathways. These results indicate that the miRNAs we identified plays a crucial regulatory role in $\mathrm{BC}$ and its immunotherapy, the basis of which might be regulating of the target genes we've identified. Therefore, these miRNAs are highly possible to be a therapeutic target for BC, which provided a direction for immunotherapy of BC at the miRNA level.

The expression of PD-L1 is of great importance in tumor immune escape [15]. We analyzed the correlation between PD-L1 expression and cancer pathway activity. It was found that the expression level of PD-L1 was positively correlated with the activities of 11 cancer pathways (NOD-like receptor, Toll-like receptor, NF-KB, JAK-STAT, TNF, apoptosis, HIF-1, VEGF, ErbB and p53 pathway). We have already found these cancer pathways were positively correlated with the degree of immune cell infiltration and their immunogenicity, indicating the activation of these cancer pathways will strengthen the immunogenicity in breast cancer. However, the expression level of PD-L1 was negatively correlated with the activity of NOTCH pathway. Combined with our finding that inhibition of Notch pathway activity enhances the immunogenicity of BC, we assume that the increased activity of NOD-like receptor, Toll-like receptor, NF-KB, JAK-STAT, TNF, cell apoptosis, HIF-1, VEGF, ErbB, p53 pathway activity, and the inhibited activity of Notch pathway might be more suitable for immunotherapy, thereby make the PD-1 or PD-L1 blocking therapy more efficient.

To summarize, we have identified the molecules related to the immunogenicity of $B C$ from different levels including cancer pathway, protein, miRNA and gene, which has provided new basis and different targets for immunotherapy of BC. However, the verification of related molecules in molecular biology level and in clinical trials still needs further research and discussion.

\section{Declarations}

\section{Funding}

Not applicable

\section{Conflicts of interest/Competing interests}

The authors declare that they have no conflicts of interest. 
Availability of data and material

The data and materials in the current study are available from the corresponding author on reasonable request.

\section{Code availability}

Not applicable

\section{Authors' contributions}

XW: Conceptualization; SX, LH, LW: Data collection; XW, SX, LH, LW: Data analysis; XW: Draft; WW, SX, LH, LW, NW: Writingreview \& editing.

\section{Ethics approval}

Not applicable

\section{Consent to participate}

Not applicable

\section{Consent for publication}

Not applicable

\section{References}

1. Siegel RL, Miller KD, Fuchs HE, et al. Cancer statistics, 2021. CA Cancer J Clin. 2021; https://doi.org/10.3322/caac.21654

2. Bochner BH. Re: Atezolizumab in Patients with Locally Advanced and Metastatic Urothelial Carcinoma who have Progressed Following Treatment with Platinum-based Chemotherapy: A Single-arm, Multicenter, Phase 2 Trial. Eur Urol. 2017;71(2):299-300.

3. Luen SJ, Salgado R, Fox S, et al. Tumour-infiltrating lymphocytes in advanced HER2-positive breast cancer treated with pertuzumab or placebo in addition to trastuzumab and docetaxel: a retrospective analysis of the CLEOPATRA study. Lancet Oncol, 2017;18(1):52-62.

4. US Food \& Drug Administration. Hematology/oncology (cancer) approvals \& safety notifications. 2018. https://www.fda.gov/drugs/informationondrugs/approveddrugs/ucm279174.htm. Accessed August 11, 2018.

5. Braun DA, Burke KP and Van Allen EM. Genomic Approaches to Understanding Response and Resistance to Immunotherapy. Clin Cancer Res. 2016;22(23):5642-50.

6. Mahoney KM, Atkins MB. Prognostic and predictive markers for the new immunotherapies. Oncology (Williston Park). 2014;28(suppl 3):39-48.

7. Khunger M, Hernandez AV, Pasupuleti V, et al. Programmed cell death 1 (PD-1) ligand (PD-L1) expression in solid tumors as a predictive biomarker of benefit from PD-1/PD-L1 axis inhibitors: a systematic review and meta-analysis. JCO Precis Oncol. 2017;1:1-15.

8. Le DT, Uram JN, Wang H, et al. PD-1 blockade in tumors with mismatch-repair deficiency. N Engl J Med. 2015;372(26):2509-20.

9. Snyder A, Makarov V, Merghoub T, et al. Genetic basis for clinical response to CTLA-4 blockade in melanoma. N Engl J Med. 2014;371(23):2189-99. 
10. Weber JS, D'Angelo SP, Minor D, et al. Nivolumab versus chemotherapy in patients with advanced melanoma who progressed after anti-CTLA-4 treatment (CheckMate 037): a randomised, controlled, open-label, phase 3 trial. Lancet Oncol. 2015;16(4):375-84.

11. Robert C, Long GV, Brady B, et al. Nivolumab in previously untreated melanoma without BRAF mutation. N Engl J Med. 2015;372(4):320-30.

12. Alexandrov LB, Nik-Zainal S, Wedge DC, et al. Signatures of mutational processes in human cancer. Nature. 2013;500(7463):415-21.

13. Pauley KM, Chan EKL. MicroRNAs and their emerging roles in immunology. Year Immunol. 2008;1143:226-39.

14. Wu CJ, Lu LF. MicroRNA in Immune Regulation. Curr Top Microbiol Immunol. 2017;410:249-67.

15. Juneja VR, McGuire KA, Manguso RT, et al. PD-L1 on tumor cells is sufficient for immune evasion in immunogenic tumors and inhibits CD8 T cell cytotoxicity. J Exp Med. 2017;214(4):895-904.

16. Otsuka A, Dreier J, Cheng PF, et al. Hedgehog pathway inhibitors promote adaptive immune responses in basal cell carcinoma. Clin Cancer Res. 2015;21(6):1289-97.

17. Zhang Q, Wang C, Liu Z, et al. Notch signal suppresses Toll-like receptor-triggered inflammatory responses in macrophages by inhibiting extracellular signal-regulated kinase 1/2-mediated nuclear factor kappaB activation. J Biol Chem, 2012;287(9): 6208-17.

18. Arulraj T, Barik D. Mathematical modeling identifies Lck as a potential mediator for PD-1 induced inhibition of early TCR signaling. PloS One. 2018;13(10):e0206232.

19. Creagh EM. Caspase crosstalk: integration of apoptotic and innate immune signalling pathways. Trends Immunol, 2014;35(12): 631-40.

20. Mocsai A, Ruland J, Tybulewicz VL. The SYK tyrosine kinase: a crucial player in diverse biological functions. Nat Rev Immunol, 2010;10(6): 387-402.

21. Wei J, Sun $\mathrm{H}$, Zhang A, et al. A novel AXL chimeric antigen receptor endows $T$ cells with anti-tumor effects against triple negative breast cancers. Cell Immunol. 2018;331:49-58.

22. Sugimoto MA, Vago JP, Teixeira MM, et al. Annexin A1 and the Resolution of Inflammation: Modulation of Neutrophil Recruitment, Apoptosis, and Clearance. J Immunol Res. 2016; https://doi.org/10.1155/2016/8239258

23. Van den Bossche J, Malissen B, Mantovani A, et al. Regulation and function of the E-cadherin/catenin complex in cells of the monocyte-macrophage lineage and DCs. Blood. 2012;119(7):1623-33.

24. Wang K, Li T, Xu C, et al. Claudin-7 downregulation induces metastasis and invasion in colorectal cancer via the promotion of epithelial-mesenchymal transition. Biochem Biophys Res Commun. 2019;508(3):797-804.

25. Tindemans I, Serafini N, Di Santo JP, et al. GATA-3 function in innate and adaptive immunity. Immunity. 2014;41(2):191206.

26. Furusawa J, Moro K, Motomura Y, et al. Critical role of p38 and GATA3 in natural helper cell function. J Immunol. 2013;191(4):1818-26.

27. Osada T, Morse MA, Hobeika A, et al. Vaccination targeting human HER3 alters the phenotype of infiltrating T cells and responses to immune checkpoint inhibition. Oncoimmunology. 2017;6(6): e1315495.

28. Schickel R, Boyerinas B, Park SM, et al. MicroRNAs: key players in the immune system, differentiation, tumorigenesis and cell death. Oncogene. 2008;27(45):5959-74.

29. Liu J, Mao Q, Liu Y, et al. Analysis of miR-205 and miR-155 expression in the blood of breast cancer patients. Chin J Cancer Res. 2013;25(1):46-54.

30. Ghisi M, Corradin A, Basso K, et al. Modulation of microRNA expression in human T-cell development: targeting of NOTCH3 by miR-150. Blood. 2011;117(26):7053-62. 
31. Si C, Yu Q and Yao Y. Effect of miR-146a-5p on proliferation and metastasis of triple-negative breast cancer via regulation of SOX5. Exp Ther Med. 2018;15(5):4515-21.

32. Mastroianni J, Stickel N, Andrlova H, et al. miR-146a Controls Immune Response in the Melanoma Microenvironment. Cancer Res. 2019;79(1):183-95.

33. Yuan X, Berg N, Lee JW, et al. MicroRNA miR-223 as regulator of innate immunity. J Leukoc Biol, 2018;04(3):515-24.

\section{Tables}

Table 1 Target Gene Prediction of 4 miRNAs

hsa-mir-155

\begin{tabular}{|lllllllll|}
\hline WWC1 & SMAD2 & JARID2 & TSHZ3 & ANKFY1 & BACH & LRRC40 & MIDN & CSNK1A1 \\
\hline CBL & TADA2B & TCF4 & ZIC3 & HIVEP2 & MY010 & PICALM & FOXK1 & CARD11 \\
\hline MEIS1 & SH3PXD2A & ZNF644 & CARHSP1 & RREB1 & MSI2 & SERTAD2 & TMEM136 & RCOR1 \\
\hline TOMM34 & HNRNPA3 & CHD8 & CEP41 & DNAJB1 & TPD52 & MYB & TMEM33 & VEZF1 \\
\hline GPM6B & PEL11 & RAB3B & ARL5B & PTAR1 & FOX03 & TRIM32 & SLC33A1 & PAXBP1 \\
\hline RCN2 & S1PR1 & PIK3CA & NOVA1 & AAK1 & ITK & CPEB4 & SIRT1 & PIK3R1 \\
\hline NFAT5 & HERC4 & RAPGEF2 & ZBTB38 & FAM135A & RAPH1 & CHD9 & INPP5D & DMTF1 \\
\hline TCF12 & TBRG1 & MBNL3 & LCORL & PRKAR1A & LNX2 & IL17RB & EEF2 & TPM1 \\
\hline ORMDL3 & CREB5 & MACC1 & CNOT6L & MDN1 & TMEM236 & MRVI1 & PHEX & LANCL3 \\
\hline GID4 & ABCF3 & UBE2K & RANGAP1 & MYLK & ARID2 & KANSL1 & EHD1 & TRPS1 \\
\hline TP53INP1 & MY01D & TLE4 & GSK3B & RAB11FIP2 & ATP6V1C1 & RICTOR & CREBRF & IKBKE \\
\hline SMAD5 & CDC73 & RAB30 & HMGCS1 & ARHGEF33 & ADAMTS5 & PKIA & WBP1L & BCAT1 \\
\hline FUBP1 & SHANK2 & GNAS & IRAK3 & DCAF17 & WNK1 & ZNF248 & ZNF699 & MY06 \\
\hline FLT1 & MMP16 & SYT6 & ATXN1L & & & & & \\
\hline
\end{tabular}

hsa-mir-150

\begin{tabular}{|lllllllll|}
\hline ADIPOR2 & HILPDA & MBD6 & STAT1 & TP53 & AIFM2 & EGR2 & NR2F2 & PDCD4 \\
GOSR1 & EPHB2 & ZBTB7A & MMP14 & GAN & VPS53 & EX05 & SRCIN1 & MTMR9 \\
\hline MKNK2 & CREBZF & COL4A4 & CYTIP & REEP4 & DAZAP2 & MYB & ENND4C & TMEM138 \\
MRPS25 & IGF1R & WDR26 & SFXN1 & GNL3L & EMP1 & STXBP5L & ORAI2 & LRRC1 \\
UBN2 & TCEANC2 & PHAX & MACC1 & KDELR1 & MLXIP & SOD2 & MRS2 & BTLA \\
PCP4L1 & SP1 & CD46 & VMP1 & ZEB1 & PRKCA & GCFC2 & XIAP & EP300 \\
TRPS1 & YRDC & INTU & CBL & NOTCH3 & SRXN1 & DHX33 & & \\
\hline
\end{tabular}

hsa-mir-146 


\begin{tabular}{|lllllllll|}
\hline MMP16 & TRAF6 & IRAK1 & SMAD4 & WASF2 & CARD10 & ERBB4 & HNRNPD & CD80 \\
TLR4 & ELAVL1 & CXCL12 & RAC1 & LAMC2 & RNF11 & PTGS2 & ROCK1 & CASP7 \\
NOS1 & SLC5A5 & COPS8 & PTGES2 & NOTCH1 & CCND2 & ZNRF3 & LRP2 & RARB \\
BCLAF1 & FANCM & NFAT5 & EGFR & FAS & & & & \\
\hline
\end{tabular}

hsa-mir-223

\begin{tabular}{|lllllllll|}
\hline RHOB & NFIA & IGF1R & SCARB1 & PTBP2 & ATM & STAT1 & EGF & E2F1 \\
ECT2 & PRDM1 & CARM1 & MAFB & ZEB1 & LMO2 & CHUK & FBXW7 & HSP90B1 \\
\hline
\end{tabular}

Figures 
TCGA
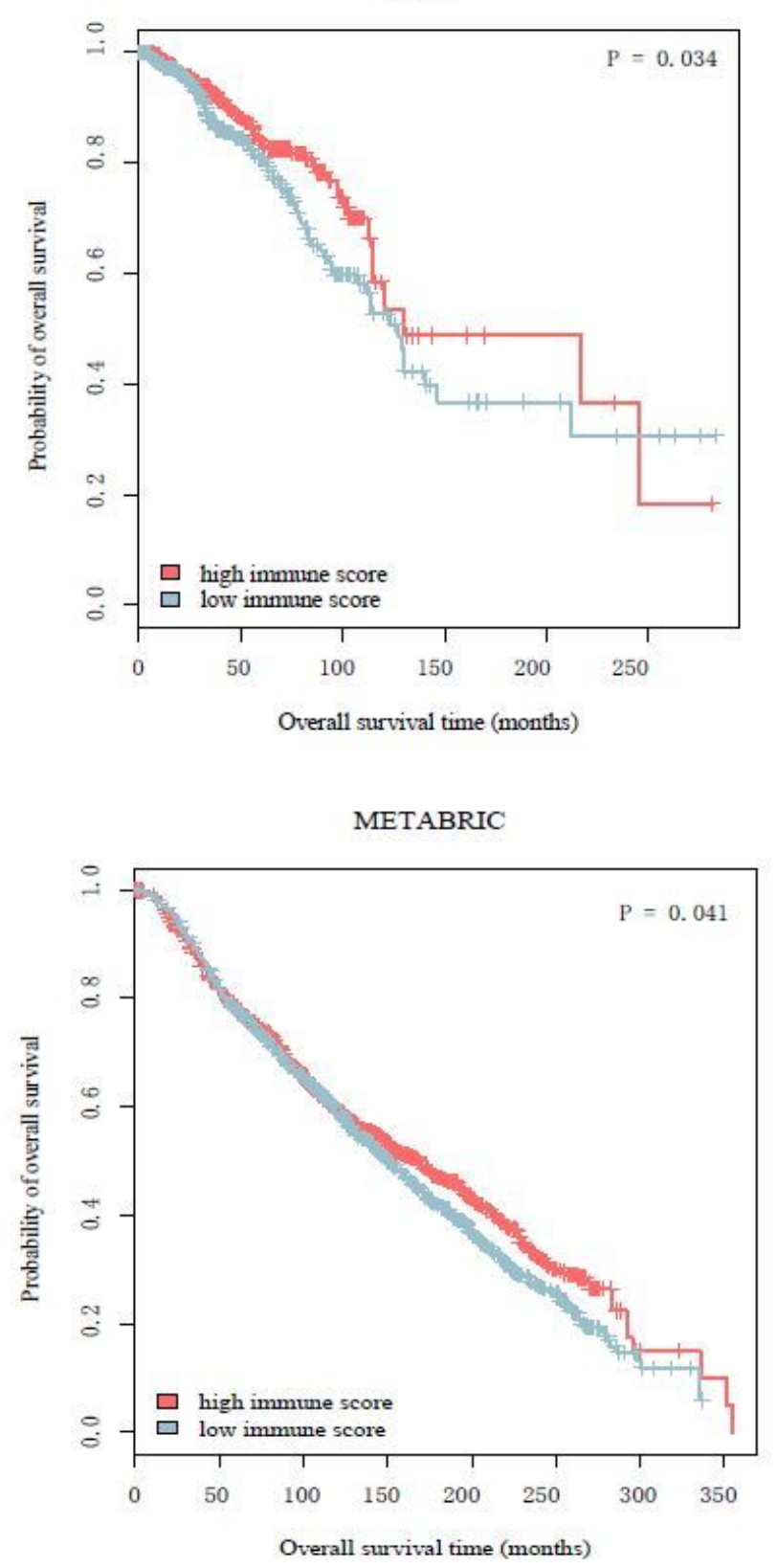

Figure 1

Kaplan-Meier survival curves in BC with high/low degree of immune cell infiltration (log-rank test, $\mathrm{P}<0.05$ ) 
A

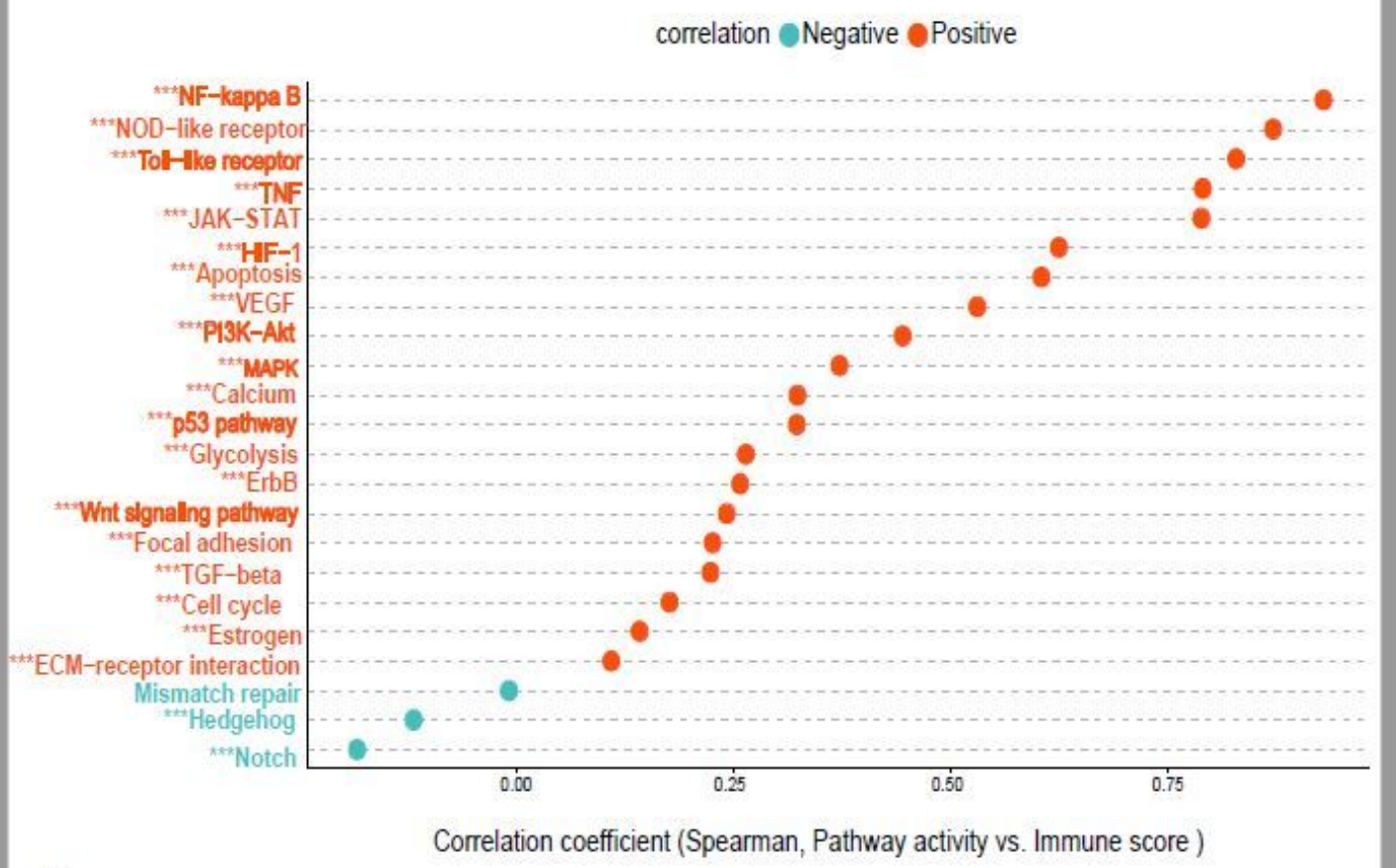

B

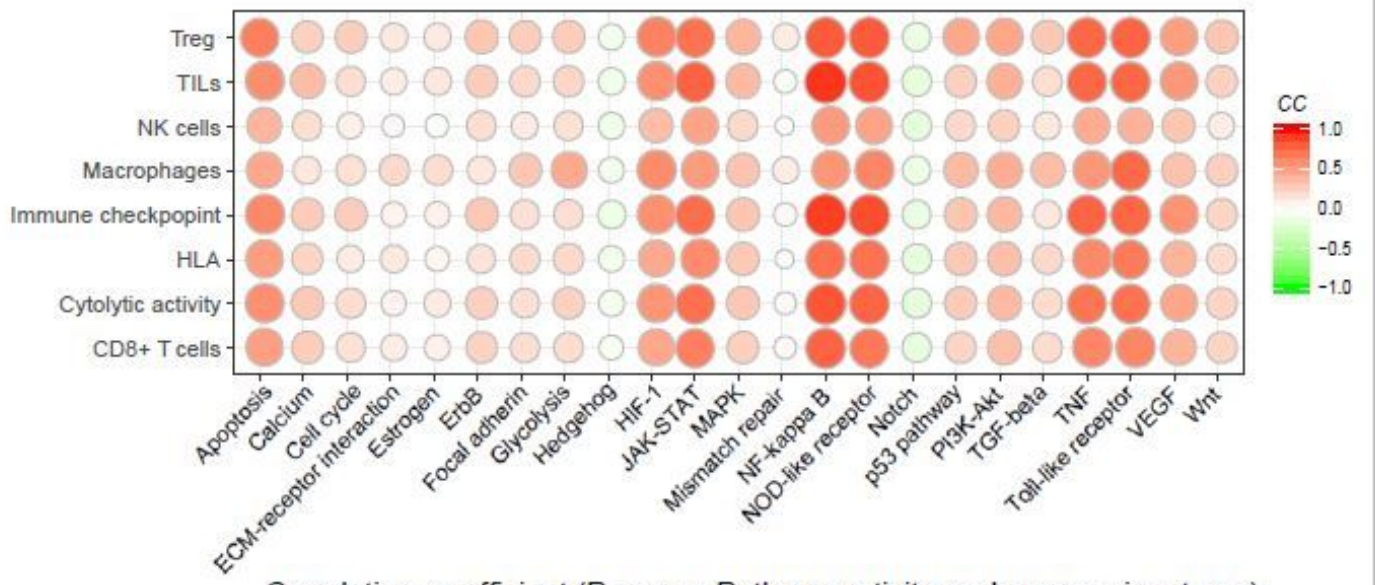

Correlation coefficient (Pearson, Pathway activity vs. Immune signatures)

Figure 2

Correlations of cancer-associated pathways with immune cell infiltration (immune score) and immune signatures in BC. a. Cancer-associated pathways that significantly correlate with immune score (Spearman's correlation test, FDR<0.05; *: P< 0.05 ; $* *: P<0.01$; ***: $P<0.001)$. b. Correlations of cancer-associated pathways with immune signatures (Pearson's correlation test, $\mathrm{FDR}<0.05)$. 


\section{A}

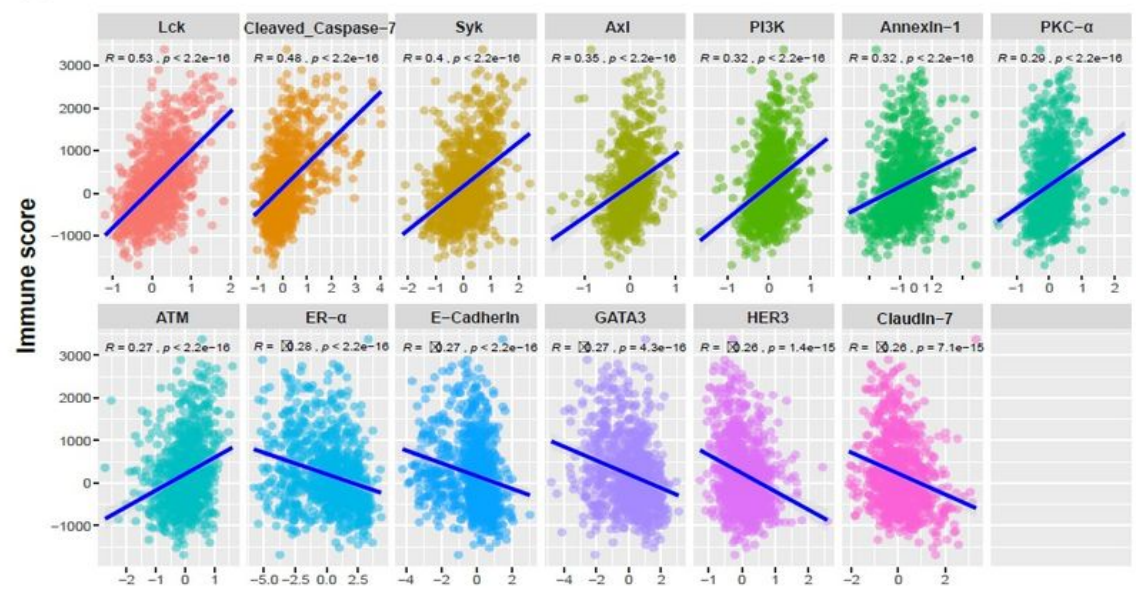

B

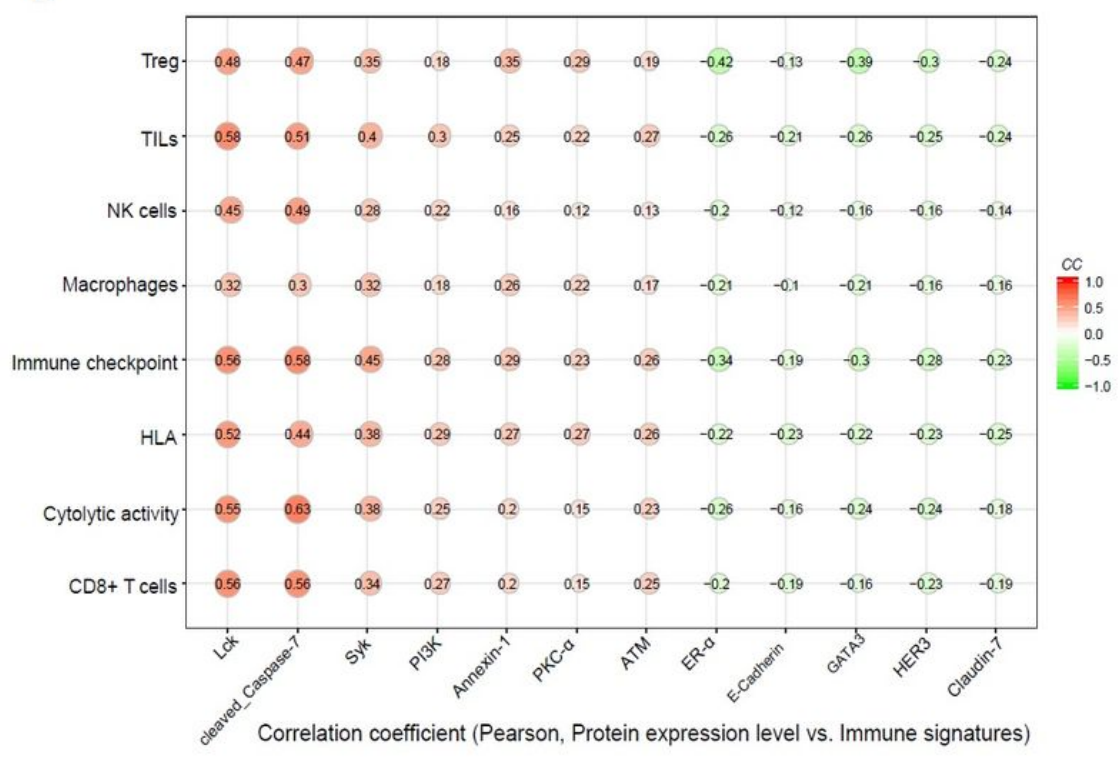

\section{Figure 3}

Correlation of proteins expression levels with immune activities in BC. a. The proteins Ick, Cleaved_Capase-7, Syk, Axl, PI3K, Annexin-1, PKC-a, and ATM expression positively correlate with immune score, and proteins ER-a, E-Cadherin, GATA3, HER3, Claudin-7 expression negatively correlate with immune score (Spearman's correlation test, FDR < 0.05). b. The Proteins Ick, Cleaved_Capase-7, Syk, PI3K, Annexin-1, PKC-a and ATM expression positively correlate with immune signatures, and the proteins ER- $a$, E-Cadherin, GATA3, HER3 and Claudin-7 expression negatively correlate with immune signatures. (Pearson's correlation test, FDR < 0.05). 

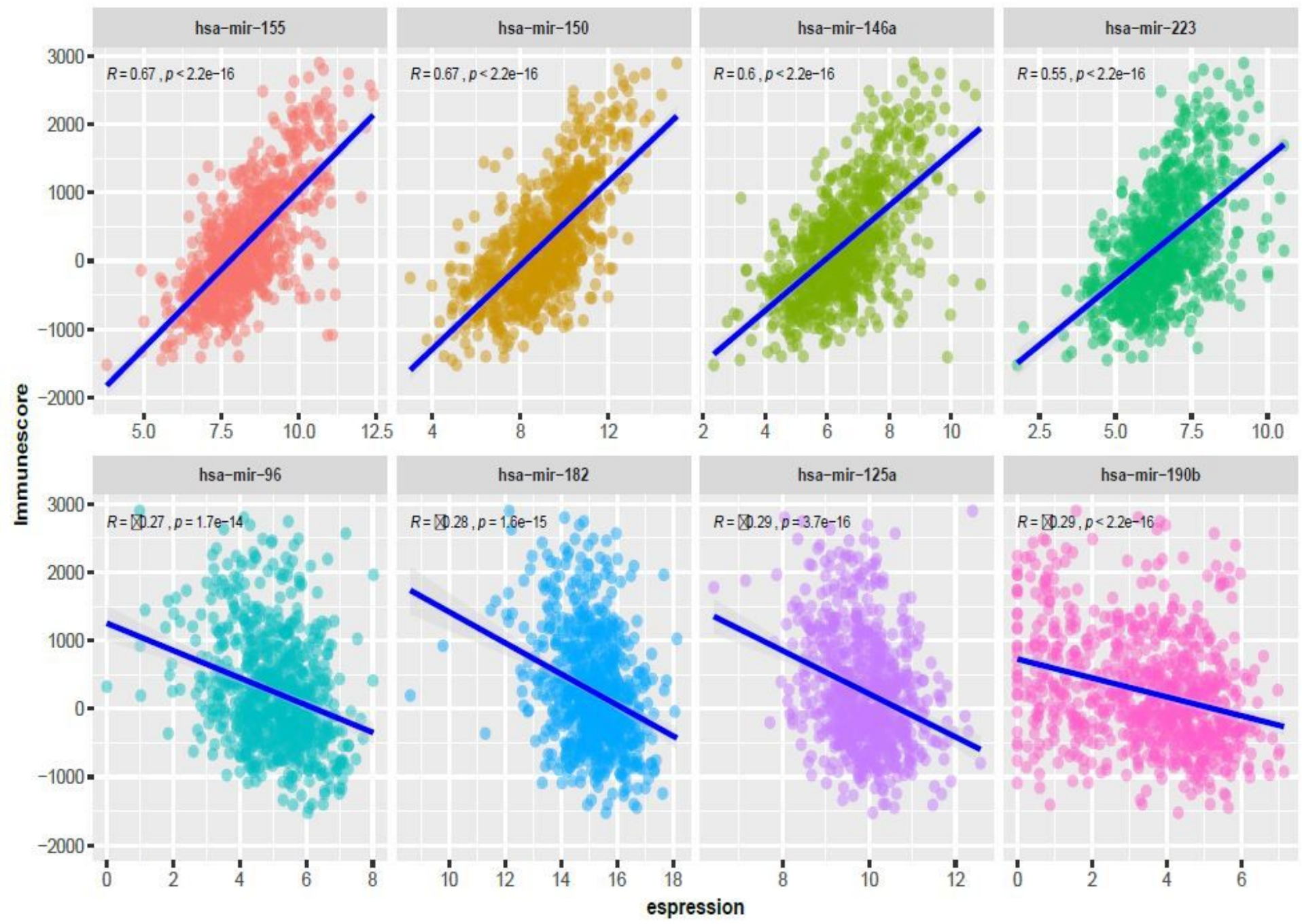

Figure 4

Correlations of miRNA expression levels with immune cell infiltration in BC (Spearman's correlation test, FDR < 0.05) 

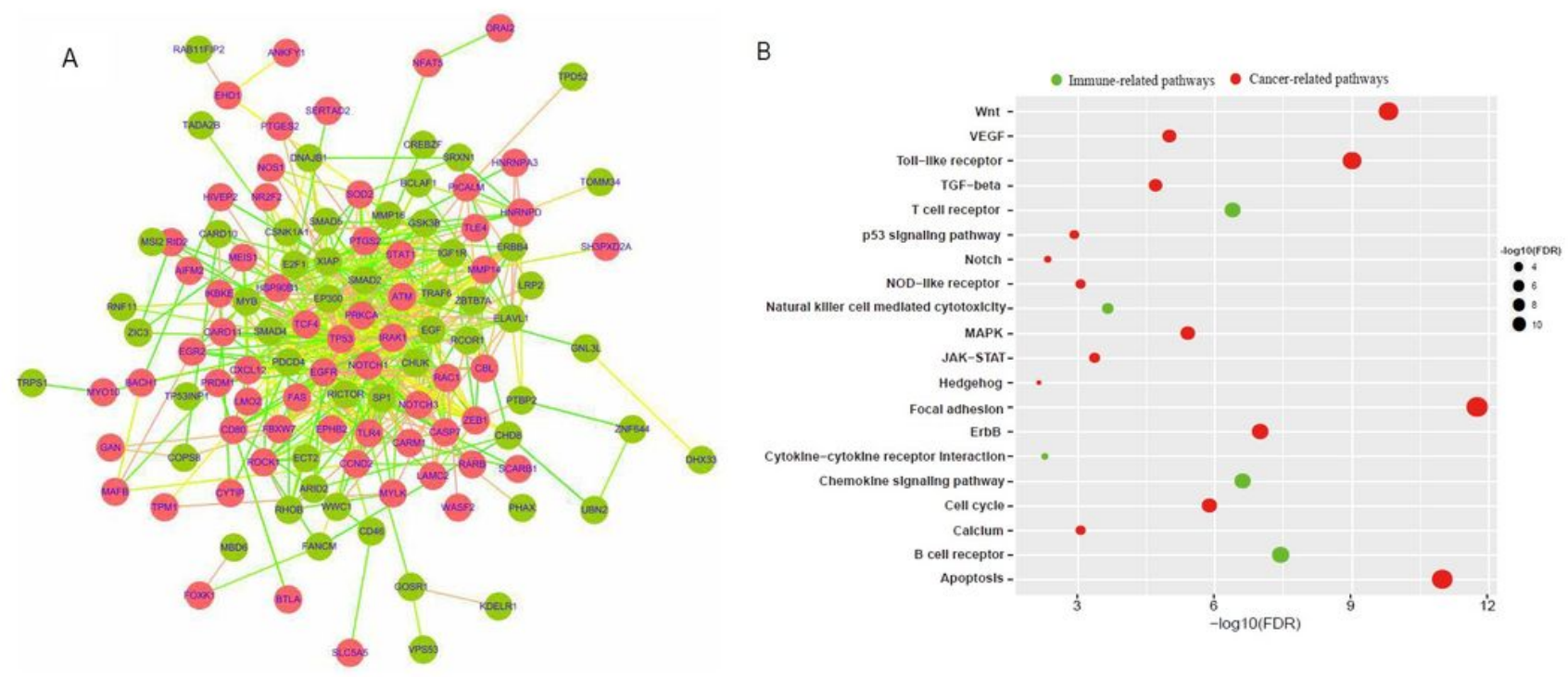

\section{Figure 5}

miRNA target gene interaction network and pathway enrichment analysis. a. miRNA-genes interaction network (plotted by Cytoscape). A red node indicates a gene whose expression is positively correlated with immune score and green indicates a gene whose expression negatively correlated with the immune score. b. KEGG pathway (cancer-related and immune-related) enrichment analysis of the genes targeted by the miRNAs whose expression is significantly associated with immune signatures. 
correlation Negative Positive

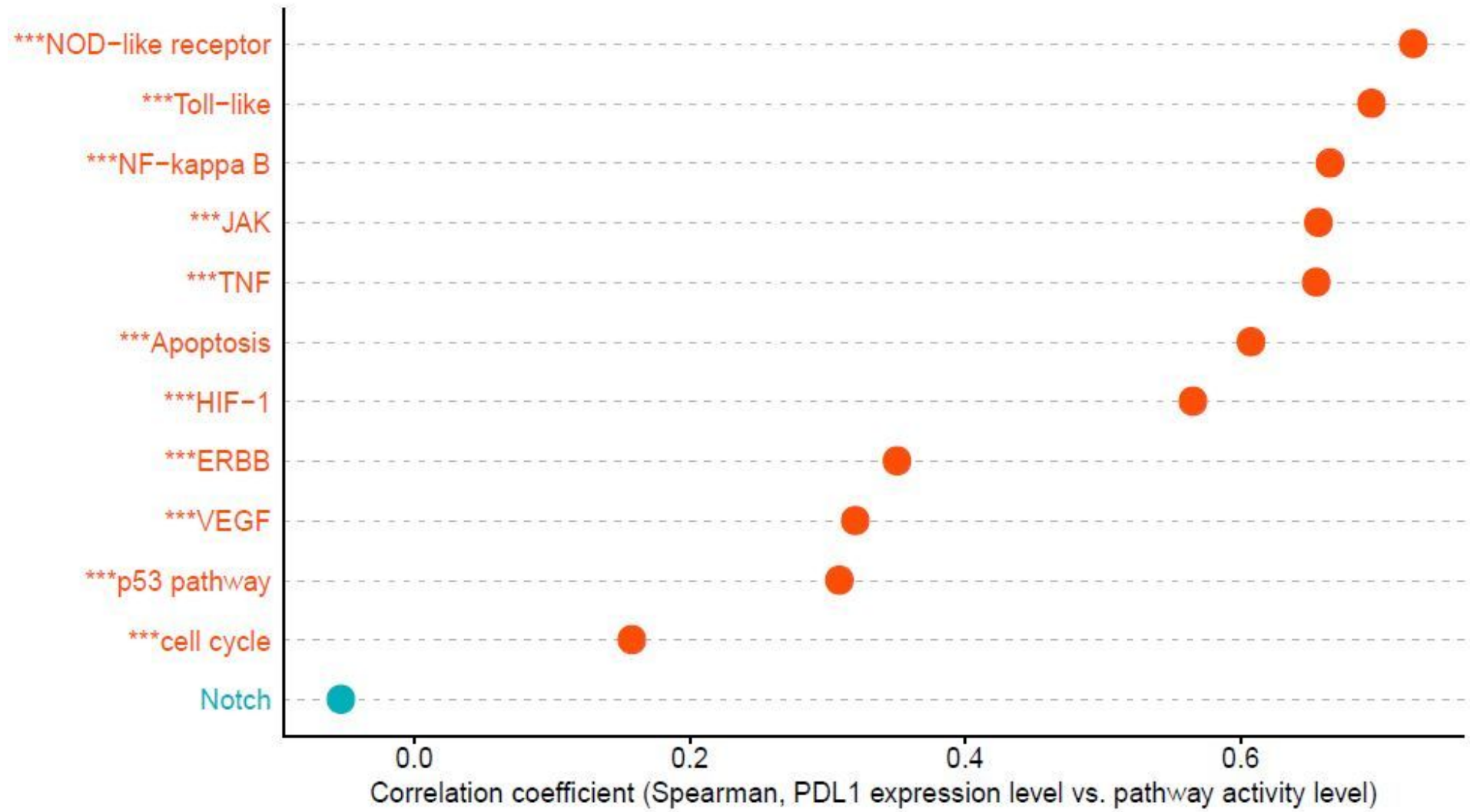

\section{Figure 6}

Correlations of PD-L1 expression level with cancer-associated pathways in BC. (Spearman's correlation test, FDR < 0.05 ; *: P $<0.05$; **: $P<0.01$; ***: $P<0.001)$. 